\title{
Scena dell'incontro tra il poeta e la Musa nel poemetto Urania di Alessandro Manzoni alla luce della tradizione classica. Analisi strutturale
}

\author{
Scene of the encounter between the poet and the Muse in the poem \\ Urania by Alessandro Manzoni in the light of the classical tradition. \\ Structural analysis
}

\author{
Alicja Raczyńska \\ Università Mikołaj Kopernik di Toruń, Polonia
}

\begin{abstract}
Riassunto: II poemetto Urania è l'ultimo lavoro neoclassico di Alessandro Manzoni, uno degli esponenti del Romanticismo italiano. L'obiettivo del mio articolo è l'analisi della scena dell'incontro fra il poeta Pindaro e la Musa Urania. Le mie ricerche mirano a riconoscere le relazioni tra la scena del poemetto e la tradizione classica dell'incontro di un poeta con una divinità ispiratrice. Le mie analisi sono ispirate alle ricerche di Tomasz Mojsik, storico e filologo classico polacco, sul ruolo delle Muse nella cultura della Grecia antica.

Parole chiave: Manzoni, Neoclassicismo, Urania, Pindaro, tradizione classica.
\end{abstract}

Abstract: The short poem Urania is the last neoclassical work of Alessandro Manzoni, one of the greatest representatives of Italian Romanticism. This paper analyses the chance meeting between Pindar and the Muse Urania. It examines whether this scene is structured in the same way as those in which chance meetings between poets and divinities take place in ancient literature. My analysis is inspired by research conducted by Tomasz Mojsik - a Polish scholar in history and classical philology — on the role of the Muses in Ancient Greek culture.

Keywords: Manzoni, Neoclassicism, Urania, Pindar, classical tradition.

Alessandro Manzoni iniziò la sua carriera letteraria con gli scritti poetici di gusto neoclassico, ispirati a Parini e a Monti'. II suo ultimo lavoro di ispirazione neoclassica è il poemetto intitolato Urania, pubblicato nel 1809, nel quale viene rappresentato il dialogo del poeta Pindaro con la Musa Urania. Secondo l'aneddoto Vincenzo Monti, leggendo quest'opera del suo giovane discepolo, avrebbe esclamato "lo vorrei finire come questo giovane ha cominciato2". "I poemetto non piacque all'autore stesso, che già nel 1809 criticò in modo aspro la propria opera in una

\footnotetext{
1 Sulle ispirazioni pariniane e montiane nelle opere giovanili di Manzoni cfr, ad esempio: Frare (1999), Finotti (1998). Le poesie di Manzoni scritte prima della conversione sono state raccolte nel volume curato da Franco Gavazzeni, edito dalla casa editrice Einaudi (Manzoni, 1992).

2 L'introduzione all'Urania in: Manzoni, 1945:16.
} 
lettera indirizzata a Claude Fauriel $^{3}$. Nel 1825 Manzoni scrisse a Tommaso Grossi definendo Urania "una vera caricatura"4. Anche secondo alcuni critici Urania non fa parte degli scritti migliori dell'autore dei Promessi Sposi. Angelo De Gubernatis ha constatato che l'ultima opera neoclassica di Manzoni "a malgrado della bellezza di alcune parti, riesce, tuttavia, un lavoro freddo e stentato [...]" (De Gubernatis, 1879: 114). Nonostante ciò il iuvenilium manzoniano ha suscitato un discreto interesse degli studiosi in quanto un'opera in cui si riflette sulla funzione educativa dell'arte e sull'azione civilizzatrice delle Muse e delle Grazie5. Nel presente articolo mi concentro sulla scena dell'incontro tra Pindaro e Urania. Le ricerche mirano ad analizzare la struttura di questa scena alla luce della tradizione classica.

A svolgere tale analisi mi ha spinto la lettura del libro di Tomasz Mojsik6 intitolato Antropologia metapoetyki. Muzy w kulturze greckiej od Homera do końca $\vee$ w. p. n. e. (Mojsik, 2011). Nel capitolo quarto dal titolo Antropologia metapoetyki - sceny inicjacji poetyckiej i płeć kulturowa $\mathrm{Muz}^{8}$ l'autore analizza, fra l'altro, le più famose scene degli incontri tra poeti e varie divinità ispiratrici nella letteratura greca. Si prendono in esame le leggende legate ad Esiodo, Archiloco, Ipponace, Eschilio, Epimenide, Omero, Stesicoro, Anacreonte, Callimaco, Platone, Pindaro ed Esopo (Mojsik, 201 1: 222-255). In base alle fonti esaminate Tomasz Mojsik ha individuato sei elementi strutturali delle suddette scene (Mojsik, 2011: 242-244). II primo elemento è il tempo dell'incontro, particolare e liminale. II poeta vede le divinità ispiratrici a mezzogiorno, di notte, nel sogno o nel tempo indeterminato. II secondo è il luogo dell'incontro. Si tratta di un posto isolato, allontanato dal mondo civilizzato, ad esempio prato, montagna, riva del mare, grotta o spazio fra le due città. II terzo è il protagonista, rappresentato di solito come un uomo giovane, immaturo, sospeso fra la civiltà e la natura. Può essere un pastore (Esiodo, Epimenide) oppure una persona di basso stato sociale (Esopo). II quarto elemento sono le divinità incontrate. Mojsik le ha divise nelle seguenti categorie: le Muse, gli esseri che hanno il contatto con la sfera soprannaturale (ad esempio le api), le personificazioni dei tratti caratteristici attribuiti all'opera letteraria dall'autore (Epimenide vede Dike ed Aletheia), le divinità legate ad un dato genere letterario, le personificazioni del genere oppure i leggendari esponenti del genere e, infine, protagonisti dell'opera lad Omero e Stesicoro sarebbe apparsa nel sogno Elena). Mojsik osserva che a volte quegli esseri immortali assumono le sembianze di qualcun altro. Le Muse che incontra Archiloco sembrano un gruppo di popolane'. Solo dopo la loro partenza, il poeta si accorge di aver parlato con le divinità. II quinto elemento è il carattere dell'incontro. Mojsik nota che l'incontro con le divinità sconvolge il poeta e cambia per sempre la sua vita come nel caso di Esiodo, umiliato dal gruppo delle Muse che deridono il suo stato sociale e si vantano del proprio sapere. Alla fine del discorso le dee gli ordinano di

\footnotetext{
3 Cfr. L'introduzione all'Urania, op. cit., Prandolini (2003: 149-150).

4 Cito da: Prandolini (2003: 150).

${ }^{5}$ Ad esempio: Accame Bobbio (1963: 28-35), Prandolini (2003:150). Gli stessi temi vennero affrontati nei poemi Musogonia di Vincenzo Monti e Grazie di Ugo Foscolo.

6 Tomasz Mojsik si è laureato in storia e in filologia classica all'Università di Varsavia, dove ha poi conseguito il dottorato in storia antica. Ora è docente e ricercatore presso l'Università di Białystok.

7 La traduzione italiana del titolo da me proposta: Antropologia della metapoetica. Muse nella cultura greca da Omero alla fine del secolo $\mathrm{V}$ a. C.

${ }^{8}$ Antropologia della metapoetica - scene dell'iniziazione poetica e il sesso culturale delle Muse.

9 La scena dell'incontro tra Archiloco e le Muse venne descritta nell'iscrizione a Paros, oggi conosciuta come l'iscrizione di Mnesiepe, cfr. Mojsik, 2011: 228.
} 
cantare gli immortali10. A volte l'incontro con le divinità provoca i cambiamenti fisici del protagonista, quali la cecità, la pazzia o la perdita della memoria (Mojsik, 201 1: 243). Tuttavia in alcune leggende citate dallo studioso polacco, l'incontro con le divinità si svolge in modo pacato. Possiamo addurre come esempi le avventure di Eschilio e Pindaro ricordate nella Pariegesi della Grecia di Pausania ${ }^{1}$. Nella prima, un giorno il giovanissimo Eschilio, addormentatosi nel campo, vide nel sogno il dio Dionisio che gli disse di scrivere le tragedie. Nel caso di Pindaro, invece, sentendosi il protagonista stanco per il caldo durante il viaggio a Tesbia, si trasse in disparte della via pubblica e si addormentò, occasione che colsero le api per mettere sulle labbra di lui i loro favi. Infine, il sesto elemento strutturale è un dono particolare ricevuto dal protagonista. Quel dono può essere un oggetto che simboleggia il nuovo stato del poeta. Ad Archiloco, ad esempio, viene donata una lira. In molte leggende i protagonisti ricevono delle capacità particolari che non avevano prima, quali la dolcezza del canto, il talento poetico oppure l'arte del bel dire. Le scene dell'incontro tra gli vomini dotti con le divinità si possono trovare anche nella letteratura latina. Tomasz Mojsik ricorda i brani degli Annali di Ennio, nei quali il poeta narra come gli sia apparsa nel sogno l'ombra di Omero (Mojsik, 2011 : 240). Possiamo anche addure come esempio l'elegia III 3 di Properzio, chiamata anche "Somnium Propertii". I primi versi del componimento rappresentano il giovane poeta che, trovandosi nelle ombre dell'Elicona, si sforza invano di scrivere un poema eroico. Ad un certo punto si accorge di essere osservato di nascosto da Apollo. II dio protettore dei poeti gli ordina di rinunciare ai versi eroici e scrivere le poesie d'amore, grazie alle quali acquisterà una grande fama.

Osserviamo ora la costruzione della scena dell'incontro tra il poeta e la divinità ispiratrice nell'Urania di Manzoni. II tempo dell'azione non è precisato, come nel passo della Teogonia che descrive la conversazione del giovane Esiodo con le Muse. II luogo dell'incontro è un posto isolato e lontano dalla gente. Pindaro, volendo sottrarsi "al volgo" (v. 90), si mette in un viaggio solitario e giunge in un bosco, rappresentato come uno spazio liminale, dove il mondo umano si scontra con il mondo divino. A volte ci vengono le Muse per dilettarsi di "I'erbe / Da mortal ombra non offese ancora" (vv. 101-102). Entrando nella misteriosa "selva" il poeta scorge un gruppo delle divinità allegoriche - l'Estro, il Diletto, il Pensamento, il Silenzio e la Gloria (vv. 104-110)12. Va notato che Manzoni situa quel luogo misterioso sul monte di Parnaso, dove, secondo le credenze dei greci antichi, si trovava una delle sedi di Apollo e delle Muse. Possiamo quindi scorgere un'affinità con le summenzionate leggende relative ad Esiodo e Properzio. L'autore della Teogonia e il famoso poeta elegiaco romano incontrarono le divinità ispiratrici ai piedi dell'Elicona, un altro monte legato al culto delle Muse e di Apollo.

\footnotetext{
10 Versi 22-24 della Teogonia; l'analisi di questo passo si trova in Mojsik, 201 1: 225-228.

"L'avventura di Eschilio viene raccontata nel libro I (Paus. 1, 21, 2). A Pindaro, invece, Pausania dedica un passo del libro IX (Paus. 9, 23, 2-3); su questi due brani cfr. Mojsik (201 1: 237, 240).

12 Luigi Russo nei suoi commenti all'Urania punta sui legami intertestuali fra il brano di Manzoni e la Basvilliana di Vincenzo Monti, dove vengono ricordate della Fame, del Bisogno e altri dolori umani alle porte di Parigi, cfr.l I commento all'Urania, vv. 103-1 10, in: Manzoni (1945: 21).
} 
Passiamo ai personaggi. II protagonista, come è stato già accennato, è Pindaro, il più grande poeta lirico della Grecia antica, vissuto negli anni 518-438 a. C.13. La sua arte inimitabile venne lodata da Orazio, il più grande lirico romano, nell'ode II 2 Pindarum quisquis studet aemulari. Pindaro fu anche stimato e ammirato da Manzoni ${ }^{14}$. Nell'Urania il famoso vate greco è presentato come un uomo giovane e ancora immaturo, quindi simile ai protagonisti delle leggende greche. Va tuttavia messo in evidenza che il Pindaro manzoniano, a differenza dai personaggi antichi, soffre a causa del suo grande peccato. Durante il suo pellegrinaggio nei santuari delle Muse omise Orcomene, un posto importante per il culto delle Grazie. Le tre ancelle di Afrodite, volendo vendicare l'offesa, aiutarono la giovane poetessa Corinna a sconfiggere Pindaro durante la gara poetica di Olimpia. II poeta si sente perso e dubita del senso della propria arte. Travolto dalle ansie e dal dolore, scappa dal mondo e giunge in una selva misteriosa, non visitata mai dai mortali. Si possono quindi, a mio parere, scorgere delle affinità fra Pindaro e Dante pellegrino del Canto I dell'Inferno. Entrambi i personaggi, perduti e infelici a causa dei loro peccati, si mettono in un cammino solitario e arrivano in un luogo liminale, dove si trova il confine tra il mondo naturale e quello soprannaturale. A questo punto occorre menzionare che i legami intertestuali fra l'Urania e la Divina Commedia sono già stati suggeriti da Guglielmo Alberti. Secondo lo studioso nel poemetto manzoniano si possono rintracciare delle reminiscenze della famosa scena dell'incontro tra Dante pellegrino e Beatrice nel Paradiso terrestre (Alberti 1969: 633).

La divinità appare al protagonista in forma umana, come le Muse nell'inscrizione che racconta la famosa avventura di Archilocho. Assumendo le sembianze della poetessa Mirtide, maestra di Pindaro e poi la sua concorrente nei certami poetici. Solo alla fine dell'incontro rivela la sua vera identità. È tuttavia sorprendente che il ruolo della dea ispiratrice e protettrice di Pindaro sia stato affidato proprio ad Urania, conosciuta come la Musa dell'astronomia. Secondo Aurelia Accame Bobbio Manzoni segue la tradizione letteraria settecentesca, la quale vedeva in Urania non solo l'ispiratrice della poesia scientifica d'argomento astronomico, ma anche il simbolo dell'Armonia cosmica (Accame, Bobbio 1963: 28). Inoltre, la studiosa nota delle affinità fra l'Urania di Manzoni e il poema Parteneide di Jens Baggesen ${ }^{15}$ che racconta il pellegrinaggio di tre belle sorelle, guidate dal giovane Norfrank, alla Jungfrau (Vergine), la cima più alta delle Alpi, dove si trova il regno di Venere Urania. Quella dea, coronata di stelle, è simbolo dell'amore per la bellezza celeste trionfante sull'amore sensuale e sulle passioni terrene. Come possiamo osservare, nell'opera del poeta danese echeggia il famoso mito platonico di Afrodite Urania, protettrice dell'amore nobile e puro ${ }^{16}$. II poemetto manzoniano fa anche pensare al

\footnotetext{
${ }^{13}$ Pindaro nacque vicino a Tebe, probabilmente in una famiglia aristocratica. Passò nella storia come autore dei quattro libri di epinici ordinati secondo la festa panellenica per la quale furono composti: Olimpiche, Pitiche, Istmiche e Nemee ; Danielewicz (2001: 85).

14 Su questo tema cfr., ad esempio, Bianco (2009: 65), Calderaro (1937: 172-177).

15 |l titolo originale del poema di Baggesen, scritto in lingua tedesca, è Parthenäis oder die Alpenraise. Esso venne tradotto in francese da Claude Fauriel. La traduzione intitolata La Parthénéide venne pubblicata nel 1810. Fu proprio Fauriel che fece conoscere a Manzoni il poema di Baggesen tra il 1806 e il 1807 (Accame Bobbio, 1963: 28-29). Alessandro Manzoni tentò di tradurre Parthenäis in italiano, ma rinunciò a questo compito. Scrisse, invece, il componimento dal titolo A Parteneide, in cui giustifica il proprio rifiuto di tradurre l'opera di Baggesen (cfr. Prandolini, 2003: 151).

16 |l concetto delle due Afroditi venne divulgato dal Simposio di Platone. Secondo filosofo la prima, chiamata Urania, è figlia di Urano e madre di Eros celeste. Protegge l'amore casto ed innocente. II suo
} 
libro secondo dei Principi della scienza nuova di Giovanni Battista Vico, intitolato Della sapienza poetica. Nella sezione dedicata alla metafisica poetica il grande filosofo settecentesco nota che le idee umane cominciarono dalle idee divine con la contemplazione del cielo ${ }^{17}$. Gli antichi auguri osservavano il firmamento celeste per conoscere la volontà degli dei. Le parti del cielo, descritte da loro con litui, venivano chiamati templa coeli. La prima Musa, definita da Omero "scienza del bene e del male", doveva essere Urania, contemplatrice del cielo al fine di prendere gli auguri, che poi passò a significare l'astronomia, mentre che Urania era considerata da alcuni la madre del mitico cantore Lino, rivale di Apollo stesso ${ }^{18}$. Prendendo tutto ciò in considerazione, possiamo quindi ipotizzare che Manzoni vide in Urania la maggiore e la più saggia delle Muse, la conoscitrice dei segreti divini, nonché simbolo dell'armonia cosmica e dell'amore per le cose sublimi. Proprio per questo motivo a lei è affidato il ruolo di protettrice del più grande lirico della Grecia antica. Una questione interessante da esaminare è anche l'iconografia di Urania nel poemetto manzoniano. Cesare Ripa nel suo lavoro intitolato Iconologia fornisce la seguente descrizione di questa Musa: "Avrà una ghirlanda di lucenti stelle. Sarà vestita di azzurro ed avrà in mano un globo rappresentante le sfere celesti19". Anche nella pittura del Seicento e del Settecento Urania viene immaginata come una donna coronata di stelle e avvolta da un manto azzurro ${ }^{20}$. Nel testo di Manzoni, invece, la Musa ha la fronte circondata dai raggi e le penne azzurre:

Tacque, e porse la cetra: indi rivolta,

Candida luce la recinse: aperte

Le azzurre penne s'agitâr sul tergo,

Mentre nel folto de la selva al guardo

Del suo Poëta s'involò [...]" (vv. 347-351)

Luigi Russo nei suoi commenti al poemetto di Manzoni osserva che Urania non assomiglia ad una divinità pagana, ma ad un convenzionale angelo della chiesa cattolica $^{21}$. Nell'ottica di tale interpretazione l'Urania, I'ultima opera manzoniana anteriore alla conversione, sarebbe quindi un preannuncio dei futuri cambiamenti nella vita dell'autore. Va riportata l'osservazione di Benedetto Prina, secondo cui nell'Urania "risuonano, per così dire, le prime armonie del poeta cristiano" (Prina, 1874: 14).

L'incontro con Urania cambia per sempre la vita di Pindaro. II poeta, credendo di vedere davanti a sé Mirtide, esprime il suo rammarico per la sconfitta durante la gara poetica. Non capisce perché proprio a lui, che ha sempre venerato le Muse, è capitata tale disgrazia. La Musa gli rivela nel suo canto i segreti divini, raccontandogli che nei tempi in cui la terra era abitata dalla gente rozza e incolta, Giove decise di mandare sulla terra le Virtù. Siccome esse non riuscirono a strappare

contrario è Afrodite Pandemia ("volgare"), figlia di Zeus e Dione, dea dell'amore carnale e madre di Eros volgare, cfr. Platon (2008: 150-151).

17 Giovanni Battista Vico, Principi di scienza nova, Libro secondo Della Sapienza poetica, Sezione prima - Metafisica poetica, Capitolo II Corollari d'intorno agli aspetti principali di questa scienza, capoverso 391. Ho consultato l'edizione Vico, 1992: 153-154.

18 Cfr. Paus. 9, 29, 6. Mi riferisco all'edizione: Pauzaniasz, 2005: 295.

19 Ripa (1766: 195).

20 Ad esempio Simon Vouet, "Muse Urania e Calliope"; Pierre Mignard, "Urania, Calliope e Tersicore"; Louis de Boullogne, "Urania e Melpomene".

${ }^{21}$ Cfr. il commento all'Urania, v. 349, in: Manzoni (1945: 29). 
I'umanità dal governo delle Furie, il re degli dei decise allora di inviare sulla terra le Muse e le Grazie che riuscirono a civilizzare ed educare gli uomini bestiali. Mentre le Muse insegnarono la poesia e il canto, le Grazie, invece, donarono l'arte di dilettare e persuadere 22 . Pindaro, anche se protetto dalla Musa Urania, subì una sconfitta dolorosa per aver offeso le ancelle di Afrodite. Urania spiega al suo alunno che il potere di quelle dee non va sottovalutato, perché senza il loro aiuto perfino gli immortali non sono "usi / Mover mai danza o moderar convito" (vv. 326-327). Nessun poeta può piacere alla gente non godendo il favore delle Grazie. Pindaro deve quindi placare l'ira delle divinità con i voti, giacché solo dopo aver ottenuto il loro perdono potrà diventare il più grande dei poeti, inimitabile e invincibile nelle gare poetiche.

Una questione interessante da esaminare è il comportamento di Urania durante l'incontro con Pindaro. La Musa, diversamente dalle divinità ispiratrici nella letteratura antica, viene presentata come una consolatrice, ed è paragonata da Manzoni alla madre che soccorre il bambino caduto:
Come la madre al fantolin caduto,
Mentre lieto al suo piè movea tumulto,
Che guäta impaurito e già sul ciglio
Turgida appar la lagrimetta, ed ella
Nel suo trepido cor contiene il grido,
E blandamente gli sorride in volto
Perch'ei non pianga [...] (vv. 135-141)

Inoltre, Urania è anche una salvatrice, visto che libera Pindaro dalla condizione del peccato e gli mostra la via alla perfezione spirituale. Sulla scia di Guglielmo Alberti (1969: 633) possiamo quindi paragonarla a Beatrice che conduce e svela al Dante pellegrino i segreti celesti.

Alla fine dell'incontro Urania, prima di rivelare la sua vera identità, offre a Pindaro un dono prezioso. Gli consegna la lira che sarà sempre compagna del suo canto. Questo strumento, attributo di Apollo, Ermete, Orfeo e Muse, simboleggia la poesia lirica: il protagonista riceve quindi un oggetto che è un segno del suo nuovo stato e della futura fama.

La scena dell'incontro tra il poeta e la Musa nell'Urania è strutturata come quelle presenti nella letteratura antica. Si svolge in un tempo indeterminato, in un luogo Iontano dalla civiltà e liminale. Il protagonista, un uomo giovane e ancora immaturo, incontra la divinità ispiratrice che all'inizio cela la sua vera identità. Durante quell' insolito incontro, Pindaro conosce i segreti divini e riceve non solo i consigli preziosi per la sua futura produzione poetica ma anche una lira, strumento che simboleggia la poesia lirica. Va però notato che Manzoni arricchisce tuttavia la tradizione classica con le reminiscenze dantesche, giacché nel poemetto risuonano gli echi della Divina Commedia. L'autore segue anche la tradizione letteraria settecentesca, che vedeva nella Musa Urania il simbolo dell'Armonia cosmica e della divina sapienza.

\footnotetext{
22 „E già de' lieti / Principij in cor secure, il plettro e l'arte / Sacra del plettro ai figli lor le Muse / Donâr, le Grazie il dilettar donaro / E il suäder potente", vv. 276-279.
} 


\section{Bibliografia}

Testi:

MANZONI, A. (1945): Liriche e tragedie. L. RUSSO (a cura di). Firenze: Sansoni.

- (1992): Poesie prima della conversione. F. GAVEZZANI (a cura di). Torino: Einaudi.

PAUZANIASZ (2005): Wędrówki po Helladzie. U stóp Apollina. H. PODBIELSKI (trad.). Wrocław: Ossolineum-DeAgostini.

PLATON (2008): Obrona Sokratesa. Krition. Uczta. T. WITWICKI (trad.). Warszawa: Hachette.

RIPA, C. (1766): Iconologia. Perugia: Stamperia di Piergiovanni Costantini.

VICO, G. B. (1992): Principi di scienza nuova. Milano: Mondadori.

Studi:

ALBERTI, G. (1969): "Alessandro Manzoni". E. CECCHI e N. SAPEGNO (a cura di): Storia della letteratura italiana. L'Ottocento, vol. VII. Milano: Garzanti, pp. 621-745.

ACCAME BOBBIO, A. (1963): La formazione del linguaggio lirico manzoniano, Roma: Edizioni di storia e letteratura.

BIANCO, G. (2009): Francesco De Sanctis. Cultura classica e critica letteraria, Napoli: Alfredo Guida Editore.

CALDERARO, G. (1937): Alessandro Manzoni e il mondo latino e greco, Firenze: La Nuova Italia.

DANIELEWICZ, J. (2001): "Wstęp". J. DANIELEWICZ (a cura di): Liryka starożyłnej Grecji. Warszawa - Poznań: PWN, pp. 12-148.

DE GUBERNATIS, A. (1879): Alessandro Manzoni. Studio biografico, Firenze: Successori Le Monnier.

FINOTTI, F. (1998): "Il «sublime patetico» del Monti". Lettere Italiane, vol. LX, n. 4, pp. 528-553.

FRARE, P. (1999): "Dalla «splendida bile» alla «socratica ironia»: Parini e Manzoni". B. MARTINELLI, C. ANNONI e G. LANGELLA (a cura di): Le buone dottrine e le buone lettere. Brescia per il bicentinario della morte di Giuseppe Parini, 17-19 novembre 1999, Milano: Vita e Pensiero, pp. 229: 555.

MOJSIK, T. (2011): Antropologia metapoetyki. Muzy w kulturze greckiej od Homera do końca $\vee$ w. p. n. e. Warszawa: Wydawnictwo Meriton.

PRANDOLINI, G. (2003): "Alessandro Manzoni. Mitologia come idolatria". R. BERTAZZOLI (a cura di): Dal neoclassicismo al decadentismo, vol. III: II mito nella letteratura italiana [a cura di P. GIBELLINI]. Brescia: Morcelliana, pp. 134-157.

PRINA, B. (1874): Alessandro Manzoni. Studio biografico e critico. Milano: Fratelli Rechiedei Editori. 